\title{
ASSOCIATION OF KI-67 VALUES AND PROGNOSTIC FACTORS IN BREAST CANCER
}

Maria Fernanda de Anhaia Arrieira', Fabio Postiglione Mansani', Mario Rodrigues Montemor Netto', Marina Besati Bertucci', Ruffo Freitas Junior ${ }^{2}$

'Universidade Estadual De Ponta Grossa - Ponta Grossa (PR), Brazil.

${ }^{2}$ Centro Avançado de Diagnóstico da Mama, Universidade Federal de Goiás - Goiânia (GO), Brazil.

Introduction: This study observed the relation of ki-67 values with prognostic factors and sought which cutoff points would be the best predictors for tumor staging. Objectives: Find which cut-off points for ki-67 would be the best predictors of tumor staging in breast cancer. Methods: The medical records of patients diagnosed with breast cancer from Instituto Sul Paranaense de Oncologia (Ponta Grossa - PR) from 2016 to 2017 were analyzed, and statistical tests were performed to evaluate the relationship between ki-67 and prognostic factors in order to find the cutoff point with better sensitivity and specificity in the characterization of tumor staging. Results: The highest values of ki-67 were found in younger patients and histological grade III tumors. The 30\% ki-67 cutoff point obtained $64.06 \%$ sensitivity and $62.67 \%$ specificity in differentiating tumors with lymph node metastasis $(\mathrm{N}+$ ) or without lymph node metastasis (N0). The cutoff point of $40 \%$ obtained sensitivity of $74.3 \%$ and specificity of $70 \%$ in the prediction of early or advanced tumors. Ki- 67 values were positively associated with prognostic factors such as histological grade, molecular profile, age, tumor size and lymph node involvement. Conclusions: The level of Ki-67 positivity is directly correlated with prognostic factors in breast cancer. Rates above $30 \%$ demonstrated a high correlation with lymph node involvement. The lack of studies in the literature that used the same methodology to calculate the sensitivity of ki-67 cutoff points in the prediction of lymph node metastasis was a differential point in this study, which may bring new perspectives for the use of this marker in the clinical and therapeutic orientation of breast cancer, requiring new studies with larger sampling to confirm the results found. 